\title{
COVID-19 and the next influenza season
}

\author{
Benjamin D. Singer \\ Department of Medicine, Division of Pulmonary and Critical Care; Department of Biochemistry and Molecular Genetics, Simpson Querrey Center for Epigenetics, \\ Northwestern University Feinberg School of Medicine, Simpson Querrey, $5^{\text {th }}$ Floor, Chicago, IL 60611 USA. \\ Email: benjamin-singer@northwestern.edu
}

Even in non-pandemic years, influenza and other etiologies of pneumonia represent the eighth leading cause of death in the United States, and respiratory viruses are the most commonly identified pathogens among hospitalized patients with community-acquired pneumonia (1). Epidemics of seasonal influenza occur on an annual basis. In the United States, the 2019-2020 seasonal influenza epidemic resulted in tens of millions of cases-the majority of which occurred before the coronavirus disease 2019 (COVID-19) pandemic surged. Now, COVID-19, caused by the novel severe acute respiratory syndrome coronavirus 2 (SARS-CoV-2), is an ongoing pandemic that has strained and, in some locales, overwhelmed healthcare systems. What can we expect as the COVID-19 pandemic evolves and seasonal influenza comes again? How can the epidemiology and biology of these infections inform our preparation strategies?

The last influenza pandemic, caused by the then-novel H1N1pdm09 virus, began in the spring of 2009 and caused an estimated 61 million cases, 274,000 hospitalizations, and 12,500 deaths in the United States over the following year (2). Despite its inclusion in the influenza vaccine since 2010, H1N1pdm09 circulates annually in the community and was the predominant influenza A virus strain during the 20192020 influenza epidemic. In contrast, the last human coronavirus epidemic, SARS (caused by SARS-CoV), abated because of aggressive containment procedures before a vaccine could be deployed; community transmission of SARS-CoV has not occurred since 2004. Based on the course of the COVID-19 pandemic to date and anticipated vaccine development timelines, it is clear that SARS-CoV-2 will not follow the abruptly terminating trajectory of SARS-CoV. Rather, it is likely that community transmission of SARS-CoV-2 will continue as we enter the next influenza epidemic. Several factors, at least in part, will determine the overall severity of the upcoming respiratory virus season and can inform how we prepare:

- Transmission. Influenza viruses and SARS-CoV-2 predominantly spread via respiratory droplets that are transmitted during close community contact. Consequently, social distancing policies designed to limit COVID-19 transmission are also effective against influenza (3). The corollary is that if COVID-19 cases begin to spike in the fall of 2020, re-tightening of social distancing measures could allay early spread of influenza to flatten the case-rate curves for both diseases.
- Vaccination. How closely the influenza antigens included in the annual vaccine match the viruses that circulate in the coming season determines the vaccine's efficacy. For 2019-2020, influenza vaccine effectiveness was $45 \%$ in the United States-a level comparable to years in which close vaccine antigen-circulating strain matching occurred (4). Importantly, influenza vaccine coverage for the previous season was only $45 \%$ among adults in the United States despite the recommendation for universal vaccination (5). Thus, as we await SARS-CoV-2 vaccine trials, plans to mitigate the overall burden of respiratory disease should include efforts to increase rates of vaccination against influenza, particularly among older adults, who have increased susceptibility to both influenza and COVID-19.

- Co-infection. Co-infection with another respiratory pathogen, including influenza, occurred in over $20 \%$ of SARSCoV-2-positive patients who presented with a respiratory viral syndrome early in the COVID-19 pandemic (6). The potential for co-infection carries diagnostic implications, as detection of an alternative etiology for a respiratory syndrome cannot be used to exclude COVID-19 in areas where SARS-CoV-2 testing remains limited. Clinicians may also need to modify therapeutic regimens depending on the specific co-pathogen (e.g., oseltamivir for influenza). These findings underscore the need for widespread availability of rapid diagnostics for SARS-CoV-2 and other respiratory pathogens.

- Disparities. African Americans, Latinx, and Native Americans are over-represented among COVID-19 cases and deaths (7-9). Also, disparities in influenza vaccination rates have historically existed among minority populations (10). The etiologies for healthcare disparities are complex and longstanding. The COVID-19 pandemic has highlighted these unconscionable disparities and must galvanize public health efforts aimed to limit viral transmission, increase vaccination rates, deploy rapid diagnostics, and expand other healthcare services for vulnerable populations, including communities of color, the poor, and older adults. Beyond these public health initiatives, overcoming disparities related to influenza and COVID-19-and disparities in health outcomes more generally-will require major societal change to address the longstanding racial and economic inequities that impact comorbidities, infection risk, and access to timely, high-quality healthcare. 
Humans have suffered from influenza for millennia, and we can expect that the new reality of COVID-19 will only complicate the next influenza season. Measures to reduce the overall burden of respiratory viral infection-including social distancing, increased vaccination rates, availability of diagnostics, and addressing healthcare disparities-are paramount in planning for the months ahead. Moreover, careful evaluation and modification of these factors will enhance preparedness ahead of viral pandemics yet to come.

\section{REFERENCES AND NOTES}

1. S. Jain, W. H. Self, R. G. Wunderink, S. Fakhran, R. Balk, A. M. Bramley, C. Reed, C. G. Grijalva, E. J. Anderson, D. M. Courtney, J. D. Chappell, C. Qi, E. M. Hart, F. Carroll, C. Trabue, H. K. Donnelly, D. J. Williams, Y. Zhu, S. R. Arnold, K. Ampofo, G. W. Waterer, M. Levine, S. Lindstrom, J. M. Winchell, J. M. Katz, D. Erdman, E. Schneider, L. A. Hicks, J. A. McCullers, A. T. Pavia, K. M. Edwards, L. Finelli; CDC EPIC Study Team, CDC EPIC Study Team, Community-acquired pneumonia requiring hospitalization among U.S. Adults. N. Engl. J. Med. 373, 415-427 (2015). doi:10.1056/NEJMoa1500245 Medline

2. S. S. Shrestha, D. L. Swerdlow, R. H. Borse, V. S. Prabhu, L. Finelli, C. Y. Atkins, K. Owusu-Edusei, B. Bell, P. S. Mead, M. Biggerstaff, L. Brammer, H. Davidson, D. Jernigan, M. A. Jhung, L. A. Kamimoto, T. L. Merlin, M. Nowell, S. C. Redd, C. Reed, A. Schuchat, M. I. Meltzer, Estimating the burden of 2009 pandemic influenza A (H1N1) in the United States (April 2009-April 2010). Clin. Infect. Dis. 52 (Suppl 1), S75-S82 (2011). doi:10.1093/cid/ciq012 Medline

3. M. W. Fong, H. Gao, J. Y. Wong, J. Xiao, E. Y. C. Shiu, S. Ryu, B. J. Cowling Nonpharmaceutical measures for pandemic influenza in nonhealthcare settingssocial distancing measures. Emerg. Infect. Dis. 26, 976-984 (2020). doi:10.3201/eid2605.190995 Medline

4. F. S. Dawood, J. R. Chung, S. S. Kim, R. K. Zimmerman, M. P. Nowalk, M. L. Jackson, L. A. Jackson, A. S. Monto, E. T. Martin, E. A. Belongia, H. Q. McLean, M. Gaglani, K. Dunnigan, A. Foust, W. Sessions, J. DaSilva, S. Le, T. Stark, R. J. Kondor, J. R. Barnes, D. E. Wentworth, L. Brammer, A. M. Fry, M. M. Patel, B. Flannery, Interim estimates of 2019-20 seasonal influenza vaccine effectiveness - United States, February 2020. MMWR Morb. Mortal. Wkly. Rep. 69, 177-182 (2020). doi:10.15585/mmwr.mm6907al Medline

5. Centers for Disease Control and Prevention, Flu vaccination coverage, United States, 2018-19 influenza season. [cited 2020 May 1]. Available from: https://www.cdc.gov/flu/fluvaxview/coverage-1819estimates.htm

6. D. Kim, J. Quinn, B. Pinsky, N. H. Shah, I. Brown, Rates of co-infection between SARS-CoV-2 and other respiratory pathogens. JAMA 323, 2085 (2020). doi:10.1001/jama.2020.6266 Medline

7. C. W. Yancy, COVID-19 and African Americans. JAMA 323, 1891 (2020). doi:10.1001/jama.2020.6548 Medline

8. New York City Health Department, Rates of cases, hospitalizations and deaths by race/ethnicity group. [cited 2020 May 1]. Available from: https://www1.nyc.gov/assets/doh/downloads/pdf/imm/covid-19-deathsrace-ethnicity-04302020-1.pdf

9. Arizona Department of Health Services, Data dashboard: Demographics. [cited 2020 May 1]. Available from: https://www.azdhs.gov/preparedness/epidemiology-diseasecontro//infectious-disease-epidemiology/covid-19/dashboards/index.php

10. K. Fiscella, Commentary-Anatomy of racial disparity in influenza vaccination. Health Serv. Res. 40, 539-549 (2005). doi:10.1111/j.1475-6773.2005.0w372.x Medline

Published First Release 29 May 2020

10.1126/sciadv.abd0086 